\title{
Cobertura de seguro de salud en Argentina entre los años 2001 y 2014: lectura de los avances y cuestiones pendientes
}

\section{Health insurance coverage in Argentina between 2001 and 2014: Reading the progress and outstanding issues}

\author{
Roberto Ariel Abeldaño* \\ Leandro Mariano González* *
}

\section{Resumen}

En este trabajo se analizó la cobertura de seguro de salud de la población argentina entre los años 2001 y 2014. Para el análisis se utilizaron como fuentes de datos el Censo Nacional de Población y Vivienda 2001 y 2010, las Encuestas Permanentes de Hogares del período 2010-2014 y la Encuesta Nacional de Gastos de los Hogares del año 2012. Se construyeron indicadores de cobertura de seguro en la población siguiendo la propuesta de Sherri et al. (2012) y finalmente se definió un modelo multivariado para identificar los factores asociados con la cobertura de seguro en la población.

Los resultados evidencian que, si bien la cobertura de seguro de salud en el período 2001 2014 se fue incrementando, en el año 2014 aún persistían sin cobertura el 30\% de la población en los aglomerados urbanos y el 50\% de las personas en todo el país. Se puede concluir que

\footnotetext{
* Becario postdoctoral CONICET. Escuela de Salud Pública. Facultad de Ciencias Médicas. Universidad Nacional de Córdoba, Argentina. Contacto: ariabeldanho@gmail.com

** Investigador de CONICET. CIECS-CONICET. Córdoba, Argentina. Contacto: leandrogonzalez@yahoo.com.ar
} 
existen diferenciales relacionados a factores geográficos y a factores sociales respecto de la cobertura de seguro de salud.

Palabras clave: Cobertura universal de salud; Sistema de salud; Salud pública.

\begin{abstract}
This paper aims to analyze the health insurance coverage of Argentina's population between 2001 and 2014. For analysis were used the data included in the National Population and Housing Census 2001 and 2010, the Permanent Household Surveys 2010-2014 and the National Survey of Household Expenditure 2012. Indicators of insurance coverage of the population were constructed following the proposal of Sherri et al. (2012) and finally a multivariate model was defined to identify associated factors with insurance coverage of the population.
\end{abstract}

The results show that although the health insurance coverage in the period 2001-2014 was increased, in 2014 still persisted without cover $30 \%$ of the population in urban areas and $50 \%$ of people around the country. It can be concluded that there are differential related to geographical factors and social factors on coverage of health insurance.

Keywords: Universal Health Coverage; Health system; Public health.

Clasificación/Classification JEL: I18, I38

\title{
1. Introducción
}

La seguridad social comprende un conjunto de medidas previsivas dirigidas a garantizar a los habitantes de un país los medios económicos para lograr condiciones mínimas de salud y educación, así como las providencias contra algunos riesgos inherentes al ciclo vital, como enfermedad, desempleo, invalidez, vejez y algunas condiciones de vulnerabilidad socioeconómica (Fleury, 2002; Mesa-Lago, 2000; Martínez, 2001).

Como se señaló en el párrafo anterior, uno de los elementos que conforman a la seguridad social tiene que ver con el aseguramiento contra los riesgos de salud. En este sentido, en los últimos años los organismos internacionales han puesto un gran empeño dirigido a lograr la 
cobertura universal de salud (CUS) en la población, entendiendo que el acceso universal a la salud y la cobertura universal de salud implican

que todas las personas y las comunidades tengan acceso, sin discriminación alguna, a servicios de salud integrales, adecuados, oportunos, de calidad, determinados a nivel nacional, de acuerdo con las necesidades, así como a medicamentos de calidad, seguros, eficaces y asequibles, a la vez que se asegura que el uso de esos servicios no expone a los usuarios a dificultades financieras, en particular a los grupos en situación de vulnerabilidad (OPS-OMS, 2014a:2).

El acceso universal es definido como la ausencia de barreras de tipo geográfico, económico, sociocultural, de organización o de género; este podrá lograrse a través de la eliminación progresiva de los obstáculos que impiden que todas las personas utilicen servicios integrales de salud, determinados a nivel nacional y de manera equitativa (OPS-OMS, 2014b). Por su parte, la cobertura de salud puede definirse como la capacidad del sistema de salud para responder a las necesidades de la población, lo cual incluye la disponibilidad de infraestructura, recursos humanos, tecnologías de la salud, medicamentos y financiamiento (OMS, 2008).

El Informe Mundial de la Salud 2010 esbozó un marco conceptual para analizar los componentes de la cobertura universal de salud (WHO, 2010) y sugiere tres grandes dimensiones:

a) La primera hace referencia a la oferta de servicios que están cubiertos (cobertura del servicio);

b) La segunda se refiere a la proporción de los costos totales cubiertos a través de seguros $\mathrm{u}$ otros mecanismos solidarios de cobertura de riesgos (cobertura financiera); $y$

c) La tercera dimensión es la relacionada con la proporción de la población cubierta (cobertura de la población).

En este marco de análisis, la primera dimensión representa la aspiración de que todas las personas puedan obtener los servicios de salud que necesitan, mientras que la segunda representa la aspiración de garantizar que la población no sufra dificultades financieras vinculadas al pago por servicios de salud en el momento en que los necesiten. La tercera dimensión refleja la distribución de la cobertura a través de diversos subgrupos de población, lo que en su conjunto realza la importancia de lograr la equidad en la cobertura por sexos, edades, lugar de residencia, condición de migrante, origen étnico y nivel de ingresos (Sherri et al., 2012). En consecuencia, la cobertura universal de salud implica que los mecanismos de organización y financiación deben ser suficientes para cubrir a toda la población. 
En este contexto, es útil distinguir que la mejora en la accesibilidad busca eliminar gradualmente las barreras financieras a la atención médica y evitar el empobrecimiento relacionado con la salud. La accesibilidad también está relacionada a la fracción del gasto de los hogares destinada a la salud sobre el gasto total de los hogares. En este sentido, la protección financiera implica minimizar los pagos de bolsillo y la compensación por la pérdida de productividad debido a la enfermedad. Entre otras cosas, la protección financiera aborda el riesgo de empobrecimiento debido a eventos catastróficos en salud, los pagos de bolsillo y los costos de transporte para llegar a los centros de salud especialmente en las zonas rurales (Scheil-Adlung et al., 2010).

Una de las maneras de concretizar la protección financiera en la población es a través de la cobertura de seguro de salud u obra social mediante un sistema de seguro social, el cual resulta de la aplicación estratégica de una política de seguridad social al amparo de los principios de solidaridad y universalidad (Titelman y Uthoff, 2003). Esta estrategia de protección financiera se configura a través de un conjunto de transacciones mediante las cuales el pago de un monto determinado permite recibir otro pago si ocurren ciertos eventos (Sojo, 2003), cubriendo una parte de los riesgos naturales del ciclo vital relacionados a la enfermedad.

La problemática de la carencia de cobertura de obra social o plan de salud se comporta como una expresión de vulnerabilidad sanitaria en la población, ya que, de acuerdo a la literatura, existen proporciones que pueden ir desde el 10 al 40\% de la población -según la jurisdicción- que se encuentra totalmente excluida del sistema de salud, debido a que registran niveles críticos de riesgo (Castro, 2009; Golovanevsky, 2007). Algunos autores reportan que la exclusión de esta cobertura afecta principalmente a una gran proporción de la fuerza laboral no asalariada, aunque también a los trabajadores asalariados (Golovanevsky, 2007).

A partir de lo señalado en el marco de análisis de la cobertura universal de salud del World Health Report, en este trabajo se propone analizar la tercera dimensión señalada por el reporte: la cobertura de seguro de salud de la población argentina en el período 2001-2014.

\section{Metodología}

Se realizó un trabajo de nivel descriptivo en donde se triangularon diversas fuentes de datos demográficos, económicos y sanitarios, con técnicas cuantitativas. El estudio tuvo un corte transversal y se trabajó con información censal del año 2001 y encuestas de población del período 2010-2014. 
Para dar cumplimiento a los objetivos propuestos, se construyeron indicadores de cobertura de obra social o plan de salud para la Argentina, según provincias y/o aglomerados urbanos, según sexos y edades. Las fuentes de datos utilizadas en estos análisis correspondieron al Censo Nacional de Población y Vivienda (CNPV) de los años 2001 y 2010, las Encuestas Permanentes de Hogares (EPH) del período 2010-2014 y la Encuesta Nacional de Gasto de los Hogares (ENGH) del año 2012.

Para caracterizar la cobertura de seguro a través de la adscripción al sistema de salud se utilizó la siguiente pregunta, incluida en el CNPV, en las EPH y en la ENGH: "¿Está asociado a un plan de salud o a una obra social?".

En la primera etapa se hicieron análisis descriptivos de frecuencias y en una segunda etapa se definió un modelo de regresión logística para estimar la variable dicotómica cobertura de obra social: $y=0$ con cobertura; $y=1$ sin cobertura. Las variables regresoras se seleccionaron de acuerdo a la bibliografía (Frenz et al., 2013) y utilizando la técnica backward Wald: sexo, edad, estado civil, años de educación formal, condición de actividad, región y deciles de ingreso. Esto permitió calcular coeficientes de Odds Ratio de las variables, para estimar la cobertura por adscripción al sistema de salud.

En cuanto a las consideraciones éticas, todas las bases de datos utilizadas en este trabajo se encuentran en acceso libre a través del sitio web del Instituto Nacional de Estadísticas y Censos de la Argentina.

\subsection{Consideraciones sobre las fuentes de datos}

Los Censos Nacionales de Población, Hogares y Vivienda de la Argentina constituyen la fuente de datos poblacionales por excelencia en el país. Se trata de un censo con periodicidad decenal, en donde se releva información socioeconómica y sanitaria de toda la población. Las bases de datos de los censos de 2001 y 2010 fueron suministradas por el Instituto Nacional de Estadísticas y Censos (INDEC). Los datos fueron procesados a través del módulo Process del software Redatam SP, desarrollado por el CELADE.

Las Encuestas Permanentes de Hogares (EPH) de la Argentina son encuestas multipropósito que relevan información de hogares y personas sobre: características demográficas básicas, características migratorias, situación laboral, habitacional, educacional e ingresos; con el objetivo de caracterizar la situación socioeconómica de hogares e individuos. 
Su diseño consiste en una muestra probabilística estratificada en tres etapas de selección. En la primera etapa las unidades primarias de muestreo son las aglomeraciones, las unidades de segunda etapa son los radios censales y las unidades de tercera etapa son las viviendas (INDEC, 2015).

La cobertura geográfica de la EPH abarca 31 aglomerados urbanos, cubriendo todas las capitales de provincia y aglomerados urbanos de más de 100.000 habitantes. Tiene una periodicidad de relevamiento trimestral, realizándose cuatro estimaciones al año sobre el mercado del trabajo en la Argentina. Para el análisis de este trabajo se utilizaron los microdatos correspondientes a los cuatro trimestres del período comprendido entre los años 2010 y 2014, considerándose la muestra tomada para todos los aglomerados urbanos del país.

La Encuesta Nacional de Gastos de los Hogares 2012 (INDEC, 2015) se realizó en todo el país, a través de una muestra probabilística, polietápica y estratificada extraída de la Muestra Maestra Urbana de Viviendas de la República Argentina construida a partir del Censo Nacional de Población, Hogares y Viviendas 2010.

El objetivo fundamental de la ENGH 2012/13 es aportar información sobre las condiciones de vida de la población y de hogares desde el punto de vista de su participación en la distribución y en la adquisición de los bienes y servicios.

La información de gastos se obtiene usando una combinación de dos métodos de captación. Para los gastos habituales (alimentación, transporte público, cigarrillos, etc.), se solicitó a los miembros del hogar que durante la semana de la encuesta realizaran anotaciones en los cuestionarios. En el caso de otros gastos, se realizaron entrevistas en las cuales los hogares informaron por recordación los gastos efectuados durante distintos períodos de referencia (en el último mes, dos meses, seis meses o en el último año, según el tipo de gasto).

El criterio para el registro de los gastos es el de gasto adquirido, por lo que se registra el valor de los bienes y servicios de los cuales el hogar toma posesión (o adquiere el derecho a recibir) durante el período de referencia, con independencia del momento en que el hogar cancela la compra y del período durante el cual los consume. 


\section{Resultados}

\subsection{Cobertura de seguro en los censos de población y vivienda}

Los datos de los censos 2001 y 2010 fueron tomados como referencia para describir la cobertura de obra social o plan de salud, habida cuenta de que esta fuente de datos indaga en la totalidad de la población. De acuerdo a estos datos, la población que auto-reportó cobertura de seguro de salud en el año 2001 fue del 51.9\% en el país, mientras que ese porcentaje aumentó al 59.2\% en el año 2010. Sin embargo, la jurisdicción metropolitana de la ciudad de Buenos Aires experimentó una situación de aumento de la población sin cobertura de seguro de salud.

Además, en el año 2001 existieron 15 jurisdicciones que evidenciaron una tasa mayor que la del total país en la categoría "sin cobertura", afectando principalmente a las poblaciones del norte del país. Esta situación mejoró en el año 2010, ya que las tasas de falta de cobertura de seguro de salud mayores a la media nacional fue observada en 12 jurisdicciones, aún se observa una situación de desigualdad que sigue afectando a las poblaciones del norte del país, tal como se observa en el Cuadro 1.

\section{Cuadro 1}

Distribución de la población según porcentaje de cobertura de seguro de salud, por jurisdicción. CNPV 2001 y 2010. INDEC

\begin{tabular}{lcccc}
\hline \multirow{2}{*}{ Jurisdicción } & \multicolumn{2}{c}{ Año 2001 } & \multicolumn{2}{c}{ Año 2010 } \\
\cline { 2 - 5 } & $\begin{array}{c}\text { \% con } \\
\text { cobertura }\end{array}$ & $\begin{array}{c}\text { \% sin } \\
\text { cobertura }\end{array}$ & $\begin{array}{c}\text { \% con } \\
\text { cobertura }\end{array}$ & $\begin{array}{c}\text { \% sin } \\
\text { cobertura }\end{array}$ \\
\hline CABA & 73.8 & 26.2 & 66.5 & 33.5 \\
\hline Buenos Aires & 51.2 & 48.8 & 65.5 & 34.5 \\
\hline Catamarca & 55.0 & 45.0 & 58.7 & 41.3 \\
\hline Córdoba & 54.2 & 45.8 & 64.2 & 35.8 \\
\hline Corrientes & 37.9 & 62.1 & 49.4 & 50.6 \\
\hline Chaco & 34.5 & 65.5 & 35.7 & 64.3 \\
\hline Chubut & 60.5 & 39.5 & 69.3 & 30.7 \\
\hline Entre Ríos & 51.3 & 48.7 & 61.6 & 38.4 \\
\hline Formosa & 34.2 & 65.8 & 36.0 & 64.0 \\
\hline Jujuy & 45.8 & 54.2 & 51.2 & 48.8 \\
\hline La Pampa & 54.5 & 45.5 & 64.7 & 35.3 \\
\hline La Rioja & 59.2 & 40.8 & 59.4 & 40.6 \\
\hline
\end{tabular}




\begin{tabular}{lcccc}
\hline \multirow{2}{*}{ Jurisdicción } & \multicolumn{2}{c}{ Año 2001 } & \multicolumn{2}{c}{ Año 2010 } \\
\cline { 2 - 5 } & $\begin{array}{c}\text { \% con } \\
\text { cobertura }\end{array}$ & $\begin{array}{c}\text { \% sin } \\
\text { cobertura }\end{array}$ & $\begin{array}{c}\text { \% con } \\
\text { cobertura }\end{array}$ & $\begin{array}{c}\text { \% sin } \\
\text { cobertura }\end{array}$ \\
\hline Mendoza & 49.4 & 50.6 & 57.3 & 42.7 \\
\hline Misiones & 42.2 & 57.8 & 53.2 & 46.8 \\
\hline Neuquén & 51.3 & 48.7 & 63.1 & 36.9 \\
\hline Rio Negro & 50.1 & 49.9 & 61.9 & 38.1 \\
\hline Salta & 39.6 & 60.4 & 46.8 & 53.2 \\
\hline San Juan & 47.0 & 53.0 & 50.4 & 49.6 \\
\hline San Luis & 48.4 & 51.6 & 56.8 & 43.2 \\
\hline Santa Cruz & 70.8 & 29.2 & 82.5 & 17.5 \\
\hline Santa Fe & 58.0 & 42.0 & 64.9 & 35.1 \\
\hline Sgo. del Estero & 36.3 & 63.7 & 36.8 & 63.2 \\
\hline Tucumán & 51.8 & 48.2 & 58.8 & 41.2 \\
\hline T. del Fuego & 69.9 & 30.1 & 80.5 & 19.5 \\
\hline Total & $\mathbf{5 1 . 9}$ & $\mathbf{4 8 . 1}$ & $\mathbf{5 9 . 2}$ & $\mathbf{4 0 . 8}$ \\
\hline
\end{tabular}

Fuente: elaboración propia en base a datos del INDEC.

Al discriminar la cobertura de salud en el país por tipos de área de residencia, se encontró que en áreas urbanas de 2001 personas y más, la población con cobertura de seguro fue del 53.7\%, mientras que en las áreas rurales agrupadas de menos de 2000 personas, la población con cobertura fue del 45.7\%; por último, en las áreas rurales dispersas, la población con cobertura fue del 32.7\%. Esta situación mejoró en el año 2010, pues la población con cobertura en las áreas rurales agrupadas y dispersas del país ascendió hasta el 57.1\% y 46.1\% respectivamente.

\subsection{Cobertura de salud en las encuestas de hogares}

$\mathrm{Al}$ indagar la tasa de cobertura de seguro de salud a través de la Encuesta Permanente de Hogares del período 2010-2014, se observó que el 66.5\% y el 70.7\% de los encuestados reportaron adscripción a un plan de salud (Gráfico 1), quedando sin cobertura entre el 29.3\% y el $33.5 \%$ de la población.

Esto puede atribuirse a que las encuestas de hogares sólo indagan en poblaciones residentes en aglomerados urbanos, mientras que el censo indaga en la totalidad de la población. Por lo 
tanto, en estas encuestas el indicador no tiene la capacidad de captar la cobertura total de la población, fundamentalmente aquélla que reside en áreas rurales.

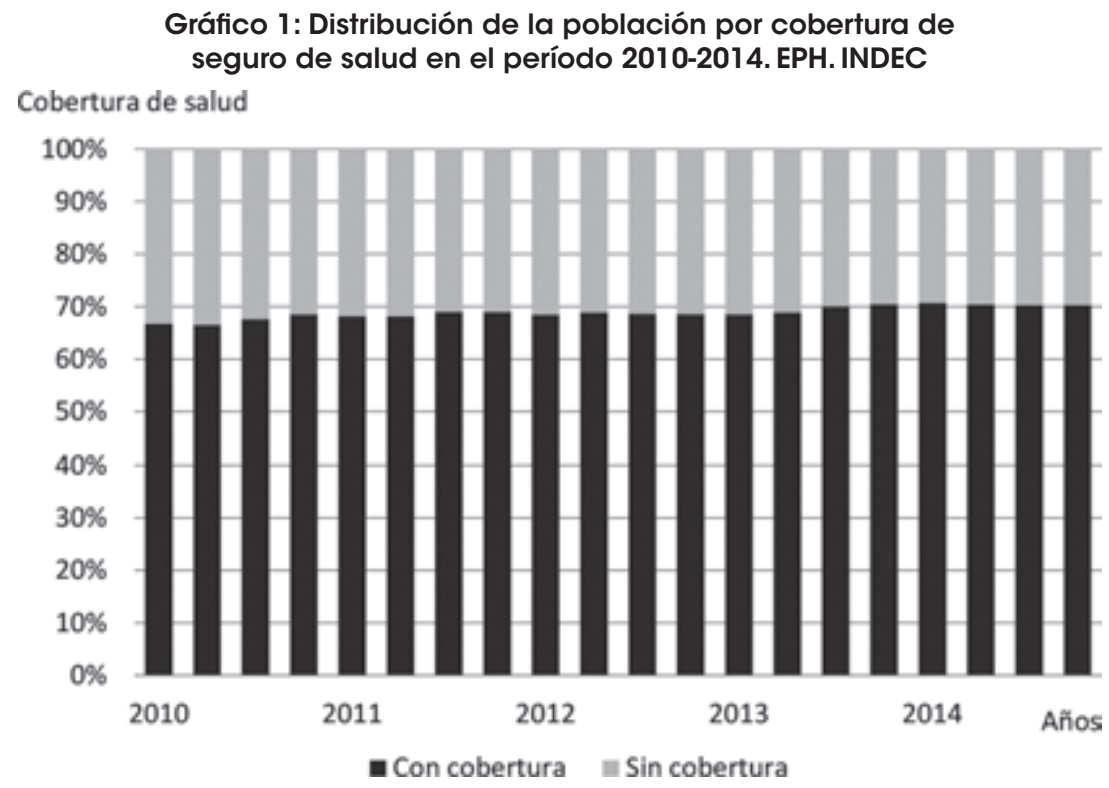

Fuente: elaboración propia en base a datos del INDEC

Alindagar la misma variable en la Encuesta Nacional de Gastos de los Hogares, la población que reportó adscripción a algún tipo de seguro de salud en el año 2012 fue equivalente al 63.7\% (Cuadro 2), teniendo así una diferencia de 4.9 puntos porcentuales entre este dato y el dato captado por al EPH en el mismo año.

En el Cuadro 2 se pueden observar los tipos de cobertura autoreportados por las personas encuestadas, teniendo en primer lugar la afiliación a obras sociales y en segundo lugar la afiliación al PAMI (Instituto Nacional de Seguridad Social para Jubilados y Pensionados, INSSJP). Esto pone de manifiesto la preponderancia del subsector de la seguridad social en el sistema de salud argentino. La afiliación a un plan de medicina prepaga ocupó el tercer lugar, con un $7.9 \%$, siendo la afliación a través de pago voluntario o a través de una obra social.

Nótese en el siguiente Cuadro que el total de las categorías de seguro de salud supera al total del $n$ de la muestra de la encuesta, debido a que una persona puede acumular más de un tipo de cobertura de manera simultánea. 


\section{Cuadro 2}

Distribución de la población por cobertura de seguro de salud en Argentina, en el año 2012. ENGH. INDEC

\begin{tabular}{lcc}
\hline \multicolumn{1}{c}{ Tipo de cobertura } & $\mathrm{n}$ & $\%$ \\
\hline Sin seguro de salud & 25941 & 36.3 \\
\hline Con seguro de salud & 45542 & 63.7 \\
\hline Obra social & 35503 & 75.0 \\
\hline PAMI & 5421 & 11.5 \\
\hline Prepaga por contratación voluntaria & 2199 & 4.6 \\
\hline Prepaga a través de obra social & 1571 & 3.3 \\
\hline Programas o planes estatales de salud & 1351 & 2.9 \\
\hline Emergencia médica & 1187 & 2.5 \\
\hline Ns/Nc & 106 & 0.2 \\
\hline Total & $\mathbf{4 7 3 3 8}$ & $\mathbf{1 0 0}$ \\
\hline
\end{tabular}

Fuente: elaboración propia en base a datos del INDEC

\subsection{Variables asociadas a la adscripción de la población con un seguro de salud}

El modelo de regresión para estimar la presencia de cobertura a través de la probabilidad de no estar adscrito al sistema de salud, permitió identificar diferencias sociales según edad, educación, ingreso, actividad, estado civil y región. La variable sexo no tuvo significación en el modelo.

El Cuadro 3 exhibe el OR de las variables del modelo en relación a las referencias. En general, a mayor edad disminuyó la chance de no adscripción, lo que puede estar motivado por la cobertura de seguro a través del Instituto Nacional de Jubilaciones y Pensiones en las personas en situación de retiro.

El estado civil de casado/a o conviviente se comportó como una categoría de riesgo respecto de soltero/a.

Los años de educación formal también disminuyeron las chances de falta de cobertura. Esta situación podría explicarse por la mejora en las condiciones laborales, salariales y de aseguramiento en personas con mayor nivel educativo, al igual que la condición de inactivo/a y ser menor de 10 años (respecto de la condición de ocupado). La población en situación de desocupación también tuvo mayor riesgo que los ocupados de no tener cobertura. 
Algunas disparidades también fueron observadas entre las distintas regiones del país. Tal como se observaba en párrafos previos, la jurisdicción del gran Buenos Aires representa un riesgo para la población residente que adolezca de cobertura de seguro de salud.

El gradiente de ingreso también se comportó como un riesgo en los deciles de ingreso más pobres, respecto de los hogares situados en deciles más ricos de ingreso total mensual del hogar.

\section{Cuadro 3}

Modelo de regresión logística para el reporte de cobertura de seguro de salud. Período 2010-2014. EPH. INDEC.

\begin{tabular}{|c|c|c|c|}
\hline \multirow{2}{*}{ Variables regresoras (referencia) } & \multirow{2}{*}{ O.R. * $p<0,05$} & \multicolumn{2}{|c|}{ I.C. $95 \%$} \\
\hline & & Inferior & Superior \\
\hline $\begin{array}{l}\text { Sexo (Varón) } \\
\text { Mujer }\end{array}$ & 0.99 _ & 0.98 & 1.00 \\
\hline Edad & $0.96^{*}$ & 0.96 & 0.96 \\
\hline $\begin{array}{l}\text { Estado civil (Soltero) } \\
\text { Casado }\end{array}$ & $1.08^{*}$ & 1.06 & 1.09 \\
\hline Años de educación formal & $0.89 *$ & 0.89 & 0.90 \\
\hline \multicolumn{4}{|l|}{ Condición de actividad (Ocupado) } \\
\hline Desocupado & $2.33^{*}$ & 2.27 & 2.39 \\
\hline Inactivo & $0.48^{*}$ & 0.47 & 0.48 \\
\hline Menor de 10 años & $0.12 *$ & 0.11 & 0.12 \\
\hline \multicolumn{4}{|l|}{ Región (GBA) } \\
\hline Noa & $0.93^{*}$ & 0.92 & 0.95 \\
\hline Nea & $1.01_{-}$ & 0.99 & 1.03 \\
\hline Cuyo & $0.94 *$ & 0.92 & 0.96 \\
\hline Pampeana & $0.71^{*}$ & 0.70 & 0.72 \\
\hline Patagónica & $0.61^{*}$ & 0.60 & 0.63 \\
\hline \multicolumn{4}{|l|}{ Deciles de ingreso (10 más rico) } \\
\hline Decil 1 -más pobre- & $16.57^{*}$ & 16.16 & 16.98 \\
\hline Decil 2 & $10.78^{*}$ & 10.53 & 11.04 \\
\hline Decil 3 & $7.22^{*}$ & 7.06 & 7.39 \\
\hline Decil 4 & $5.26^{*}$ & 5.14 & 5.38 \\
\hline Decil 5 & $3.98^{*}$ & 3.89 & 4.07 \\
\hline Decil 6 & $3.21^{*}$ & 3.14 & 3.29 \\
\hline Decil 7 & $2.58^{*}$ & 2.52 & 2.64 \\
\hline Decil 8 & $2.03^{*}$ & 1.98 & 2.08 \\
\hline Decil 9 & $1.58^{*}$ & 1.54 & 1.62 \\
\hline
\end{tabular}




\section{Conclusiones}

Los resultados relacionados a la cobertura de la población sugieren que entre el año 2001 y el año 2014 en Argentina hubo avances favorables en el aumento de la cobertura de la población; sin embargo, entre las cuestiones pendientes de resolver se encuentra la situación de que aún persisten sin cobertura 3 de cada 10 personas en los aglomerados urbanos de la Argentina, y esta relación aumenta a 5 de cada 10 personas en todo el país. Además, debe tenerse en cuenta que las encuestas de hogares no captan la población en las áreas rurales, en donde el fenómeno de carencia de cobertura de salud adquiere una mayor dimensión.

Por su parte, las diferencias en la captación de los indicadores de cobertura de la población entre las distintas fuentes de datos utilizadas en este estudio pueden asumirse como una limitación del presente estudio.

Con respecto a los factores que determinan la cobertura de la población a través del autoreporte de estar asociado a un plan de salud, se observaron diferenciales sociales, resaltando la condición de vulnerabilidad sanitaria por carencia de cobertura de salud en las personas que tienen un perfil de casados/as o unidos/as, desocupados/as, y que pertenecen a un hogar situado en los deciles de ingreso más bajos (más pobres).

Por el contrario, se puede afirmar que las personas de mayor edad que se encuentran en situación ocupacional de inactivo/a y con mayor cantidad de años de educación formal se encuentran con menores chances de no estar adscriptos a algún tipo de plan de salud. Esto puede deberse a la adscripción de esta población al sistema de protección social para jubilados y pensionados.

La falta de cobertura de seguro de salud en los hogares expone a los individuos a condiciones de vulnerabilidad a las que las poblaciones están sometidas por generaciones, restringiendo sus opciones de vida e influenciando en sus prácticas de salud, así como exponiendo a los adultos y a los niños a diversos riesgos de morbilidad y de mortalidad, de deficiencias físicas, de limitaciones a las actividades y a la participación social (Drachler, 2014).

En ese sentido, hoy, con base en la evidencia disponible, se puede hipotetizar que el aumento de la cobertura de seguro de salud en la población argentina puede deberse en parte al largo proceso de fortalecimiento de las políticas que tienen como población objetivo a los grupos más vulnerables. Entre estas políticas se puede mencionar al financiamiento del sector salud. En este sentido, los datos del Banco Mundial apuntan que en el año 2013, la Argentina 
destinó 45 billones de dólares para financiar el sistema de salud, lo que implica una inversión de 1074 dólares per cápita. El gasto que se destinó a la salud como porcentaje del gasto total fue del 32\%, mientras que el mismo organismo informa que el porcentaje del PIB destinado a la salud en Argentina en 2013 fue del 7.3\%, lo cual sugiere que Argentina destina una gran inversión orientada a la reducción de los problemas de inequidad en el financiamiento de los eventos de salud de los hogares (Banco Mundial, 2013).

Otras iniciativas que impactaron en el aumento de la cobertura de seguro son el Programa Sumar, de protección de salud para la población materno-infantil, y el Plan Remediar (Cavegnero, 2010); ambos planes se pusieron en marcha a principios de la década pasada. En el año 2004, el Gobierno nacional creó el Plan Nacer, con el objetivo de incrementar el acceso equitativo y la calidad de los servicios de salud, priorizando a las mujeres embarazadas y niños de hasta 5 años. Luego en el año 2013, a través del Programa Sumar, se amplió la población objetivo y los servicios de salud ofrecidos, y se consolidó un modelo con mayor equidad en el acceso a la salud. Cabe señalar que la Asignación Universal por Hijo (AUH) y a la Asignación Universal por Embarazo (AUE) fueron otros programas de gobierno que buscan sinergizar el efecto del Programa SUMAR en vistas al aumento de la cobertura efectiva y del fortalecimiento de la accesibilidad a la población más vulnerable (Núñez, 2015).

Por su parte, el Programa Remediar es un programa de alcance nacional creado en el año 2002, que tiene por objetivo garantizar la provisión de una canasta de medicamentos esenciales, a través del envío de botiquines y otros insumos para centros de primer nivel de atención, apuntando al fortalecimiento de la capacidad de respuesta del primer nivel de atención de todas las provincias (Programa Remediar, 2013). Este fortalecimiento de la atención primaria es una reconocida estrategia que impacta positivamente en el aumento de la cobertura efectiva de los servicios de salud de los hogares, ya que este sector es en gran parte financiado y puesto a disposición hacia la comunidad por parte del sector público (Anbari et al., 2014; Young Lee, 2014).

Sobre las mejoras en los indicadores globales de cobertura de seguro de salud en la Argentina, aunque aún persistan diferencias sustanciales en las distintas jurisdicciones, Kessler (2014) afirma que, en el período 2003-2013, el país ha experimentado un avance importante en términos de equidad, aunque persisten núcleos de exclusión estructural en referencia a quienes 
sufren ciertas 'enfermedades catastróficas' u otras graves sin adecuada cobertura o sin acceso a los servicios, quienes tienen las mayores dificultades de vivienda, los expulsados de sus tierras, los que sufren mayor violencia de distinto tipo y quienes viven en zonas relegadas, donde carencias de infraestructura y de oportunidades se retroalimentan (...) La mejora general de la situación no pareció repercutir en una disminución de las brechas de desarrollo, destacándose la particular situación desfavorable del Noroeste y, más claramente, del Noreste (340).

Fecha de recepción: 28 de junio de 2016. Fecha de aceptación: 30 de septiembre de 2016. Manejado por la A.B.C.E. 


\section{Referencias}

1. Argentina, Ministerio de Salud de la Nación. Programa Remediar. 2013. "Impacto redistributivo del programa remediar en el gasto en medicamentos”. Disponible en: www.remediar.gov.ar

2. Banco Mundial. 2013. Datos del Banco Mundial. Disponible en http://datos. bancomundial.org/indicador/SH.TBS.INCD/countries

3. Cavegnero, E. y M. Bilger. 2010. "Equity during an economic crisis: financing of the Argentine health system”. Journal of Health Economics, 29(4), 479-488.

4. Castro, J. 2009. "Hacia el aseguramiento universal en salud en el Perú". Rev Peru Med Exp Salud Pública, 26(2), 232-35.

5. Drachler, M.L., Marcos Antônio de Oliveira Lobato, José Inácio Lermen, Sandra Fagundes et al. 2014. "Desenvolvimento e validação de um índice de vulnerabilidade social aplicado a políticas públicas do SUS”. Ciência é Saúde Coletiva, 19(9):3849-3858.

6. Fleury, S. y C. Molina. 2002. “Modelos de protección social”. En: Banco Interamericano de Desarrollo, Instituto Interamericano para el Desarrollo Social (INDES): Diseño y gerencia de politicas y programas sociales. Washington: INDES.

7. Frenz, P., I. Becerra, L. Villanueva, J. Kaufman, F. Muñoz Porras et al. 2013. “Seguimiento de cobertura sanitaria universal con equidad en Chile entre 2000 y 2011 usando las encuestas CASEN". Revista Médica de Chile, 141(9),1095-1106

8. Golovanevski, L. 2007. "Vulnerabilidad y transmisión intergeneracional de la pobreza. Un abordaje cuantitativo para Argentina en el siglo XXI”. Tesis Doctoral. Doctorado en Economía. Buenos Aires: Universidad de Buenos Aires.

9. Instituto Nacional de Estadísticas y Censos (INDEC). Encuesta Permanente de Hogares. Disponible en www.indec.gov.ar

10. Kessler, G. 2014. Controversias sobre la desigualdad. Argentina, 2003-2013. Buenos Aires: Fondo de Cultura Económica.

11. Martínez, D. y M.L. Vega. 2001. La globalización gobernada. Estado, sociedad y mercado en el siglo XXI. Madrid: Editorial Tecnos (Grupo Anaya).

12. Mesa-Lago, C. 2000. Desarrollo social, Reforma del Estado y de la seguridad social al umbral del siglo XXI. Serie Políticas Sociales No 36. Santiago de Chile: CEPAL/ECLAC. 
13. Núñez, P. 2015. "Investigación sobre resultados del Plan Nacer/Programa SUMAR". Biblioteca de investigaciones y estudios del Programa Sumar. Disponible en http:// www.msal.gob.ar/sumar/index.php/institucional/biblioteca-de-investigaciones-yestudios-del-programa-sumar

14. OMS. 2008. Informe sobre la salud en el mundo 2008: la atención primaria de salud, más necesaria que nunca. Ginebra: OMS. Disponible en: http: //www.who.int/ whr/2008/08_report_es.pdf.

15. OPS-OMS. 2014a. Resolución No53 Consejo Directivo 66 Sesión del Comité Regional de la OMS para las Américas. Washington, D.C., EUA.

16. OPS-OMS. 2014b. Estrategia para el acceso universal a la salud y la cobertura universal de salud. 66a Sesión del Comité Regional de la OMS para las Américas. Washington, D.C., EUA.

17. Scheil-Adlung, X., F. Bonnet, T. Wiechers y T. Ayangbayi. 2010. "New approaches to measuring deficits in social health protection coverage in vulnerable countries". World Health Report. Background Paper No 56. WHO.

18. Sherri, H., L. Hatt, A. Leegwater, M. El-Khoury y W.Wong. 2012. Indicators for Measuring Universal Health Coverage: A Five-Country Analysis (draft). Bethesda, MD: Health Systems 20/20 project, Abt Associates Inc.

19. Sojo, A. 2003. "Vulnerabilidad social, aseguramiento y diversificación de riesgos en América Latina y el Caribe”. Revista CEPAL, 80: 121-140

20. The World Health Report (WHO).2010. Health Systems Financing: The path to universal coverage. Geneva: World Health Organization.

21. Titelman, D. y A. Uthoff. 2003. "El papel del aseguramiento en la protección social". Revista CEPAL, 81:103-122. 